\title{
Self-management interventions in COPD patients with multimorbidity
}

\author{
Lowie E.G.W. Vanfleteren (1) and Leonardo M. Fabbri (1)
}

Affiliation: COPD Center, Sahlgrenska University Hospital and Institute of Medicine, University of Gothenburg, Gothenburg, Sweden.

Correspondence: Lowie E.G.W. Vanfleteren. Sahlgrenska universitetssjukhuset, COPD Center, Vita stråket 12 , Göteborg 413 45, Sweden. E-mail: lowie.vanfleterendgu.se

@ERSpublications

Self-management interventions in patients with COPD need to address the complexity of multimorbidity and should include action plans for worsening of both COPD and common comorbidities in order to empower patients to manage their different diseases. $\mathrm{http} / / \mathrm{bit} . \mathrm{ly} / 2 \mathrm{nXSCuz}$

Cite this article as: Vanfleteren LEGW, Fabbri LM. Self-management interventions in COPD patients with multimorbidity. Eur Respir J 2019; 54: 1901850 [https://doi.org/10.1183/13993003.01850-2019].

Self-management interventions in patients with COPD have the goals of motivating, engaging and supporting patients to positively adapt their behaviours and develop skills to better manage their disease. Exacerbation action plans are a key component of COPD self-management interventions [1, 2], and self-management interventions are a key component of COPD management [3]. However, studies assessing these interventions show contradictory results, particularly depending on the primary outcome, e.g. quality of life, exacerbations, re-hospitalisations or mortality $[1,2,4]$.

Self-management interventions, including a COPD exacerbation action plan, are associated with improved health status, as measured by St George's Respiratory Questionnaire (SGRQ), and a lower probability of respiratory-related hospital admissions. Conflicting results on the incidence of exacerbation and mortality have been reported: Exploratory analyses showed a small but significantly higher respiratory-related mortality rate in patients with a high risk of exacerbation $[2,5]$.

COPD is almost invariably associated with concomitant chronic diseases, commonly termed comorbidities. A more proper term probably is multimorbidities, i.e. two or more diseases occurring in a person in whom it is difficult to identify an index disease [6]. Exacerbations of COPD should be evaluated in this context. A deterioration of respiratory symptoms in a multimorbid patient who also has COPD demands a differential diagnosis. Viral or bacterial infections, but also heart failure, coronary heart disease, pulmonary embolism and panic attacks, can be equated with an exacerbation of COPD [7].Thus, COPD is just the pulmonary component of multimorbidity, and consequently a patient with COPD should be managed comprehensively for all concomitant chronic diseases that can be identified $[3,6,8]$. However, a recent elegant and well-designed intervention aimed at comprehensively addressing the complexity of multimorbidity did not improve the quality of life of the patients studied [9].

In this issue of the European Respiratory Journal, LENFERINK et al. [10] report the results of an international randomised controlled trial intended to evaluate whether COPD patients with multimorbidities who had been trained in using a patient-tailored multidisease exacerbation action plan (figure 1) had fewer days of COPD exacerbations. Unfortunately, the study was negative for the primary outcome (number of COPD exacerbation days per patient per year) but showed a significant reduction of some relevant predefined secondary outcomes, i.e. a shorter duration per COPD exacerbation and a lower probability of at least one 

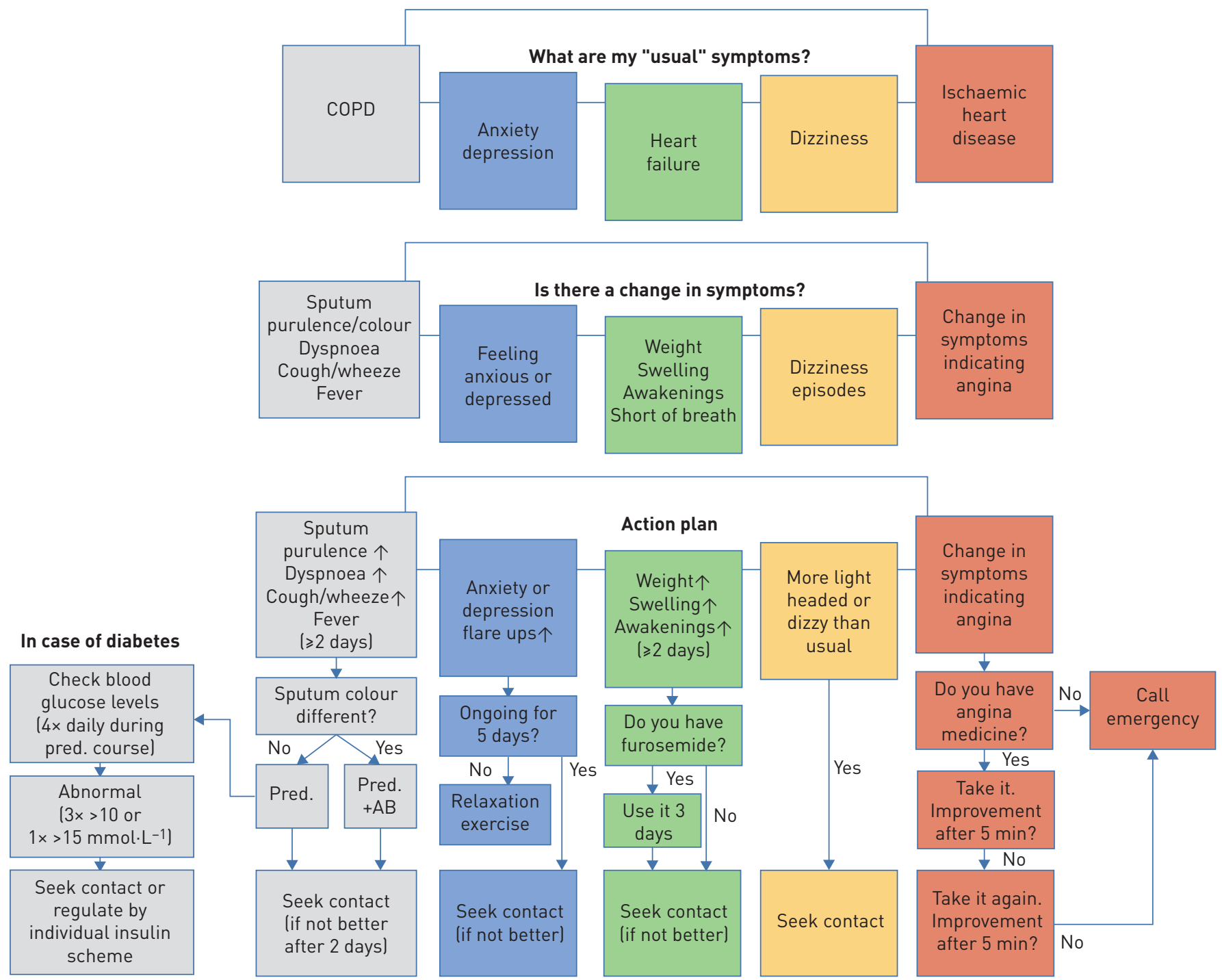

FIGURE 1 Patient-tailored action plan for COPD and some of its concomitant chronic diseases. Adapted from the supplementary material of LENFERINK et al. [10]. The action plan included a final safety check, which is not in the figure, advising patients to contact the general practitioner in case of high fever but without change in the evaluated symptoms. Pred.: prednisolone; AB: antibiotics.

respiratory-related hospitalisation, without excessive all-cause mortality. The authors conclude that, although the number of COPD exacerbation days was not significantly lower in the self-management group, their study provides new evidence that exacerbation action plans for COPD patients with multimorbidities embedded in an individualised, multifaceted self-management intervention may be an effective tool to reduce the duration of COPD exacerbations and the risk of undergoing a respiratory-related hospitalisation. Furthermore, this exacerbation action plan was safe, as it did not modify all-cause mortality. The authors recommend that the implementation of a successful self-management intervention for patients with COPD and multimorbidities requires a patient-tailored approach, i.e. one that includes an individual assessment of COPD and multimorbidities, information on self-management, daily self-monitoring of symptoms, COPD exacerbation action plans, and support from a group case manager for feedback and motivation.

There could be several reasons why the study was negative for the primary outcome. First, it is unclear why a study that selected patients with COPD and multimorbidity was powered primarily on exacerbations. Obviously, exacerbations are an important feature of COPD and multimorbidity, but they occur in only a fraction of COPD patients. In fact, almost one-third of the patients had no exacerbations during the study [3]. Two recent self-management studies conducted in COPD patients included those with a history of exacerbations in the previous year and/or low lung function [11,12] and one of the studies [12] included patients with multimorbidity. Although both studies were also negative for the primary outcome, they showed a reduction in hospital admissions and mortality [11, 12]. Almost all 
pharmacological randomised controlled trials powered on exacerbations include patients selected because of a history of both frequent exacerbations and low lung function. If the current study had been restricted to patients with not only frequent exacerbations (at least two moderate and/or one or more severe exacerbations in the previous year) but also with low lung function (forced expiratory volume in $1 \mathrm{~s}<50 \%$ pred), it might have shown more positive results for the primary outcome.

A recent Cochrane review [2] of the efficacy and safety of self-management plans for COPD, written by some of the authors of the LENFERINK et al. [10] study, underlined the consistent effect of self-management plans on health status. Both COPD Assessment Test and the Chronic Respiratory Disease Questionnaire were administered [13], and quality of life was a secondary outcome. Unfortunately, the effect of the self-management plan was arithmetically superior to usual care, but the difference was not statistically significant. These results, taken together with similar trends in the primary outcome (number of COPD exacerbation days and severity per exacerbation day), further suggest that this study [10] might have been underpowered. In fact, according to the protocol [13], 150 patients per arm were expected, but only 102 and 99 in the self-management arm and the usual care arm, respectively, were recruited.

Self-management plans have several different aims, including improved adherence to treatment, which is critical for successful disease management, particularly if the patient is elderly and has multimorbidities [14]. Although the authors of the current study did not measure adherence to COPD treatment and treatment of multimorbidities, the differences between patients with improved compared to poor adherence might explain the lack of effect.

The authors' self-management interventions did not include a case manager for each patient. Having individual case managers would probably increase the efficacy of the action plans. Most likely, patients with complex multimorbidity would benefit from the ability to consult a medical professional in case of doubt about the plan, worsening symptoms, decisions about medication, or referral for an unscheduled visit to a physician, an emergency room visit, or hospital admission.

The lack of effect of self-management interventions on multimorbidities is also of some concern but might be related to the fact that patients were selected primarily for COPD as an index disease. Thus, $100 \%$ of patients had COPD whereas not all had the same concomitant chronic diseases. Selecting a more homogeneous group of patients with, for example, COPD, hypertension, ischaemic heart disease, congestive heart failure and symptoms of anxiety or depression (a common phenotype of multimorbidity), possibly hospitalised after an acute severe exacerbation of one of the components of the multimorbidity, might show a better effect of self-management.

The significant reduction in duration of exacerbations is certainly an interesting finding. It would be useful to confirm whether, as suggested by the authors, this was due to the patients' early recognition of the exacerbation or to an improved treatment. The observation that more patients in the self-management group took oral steroids within 2 days of the onset of a COPD exacerbation suggests that patients learned to identify an exacerbation, to treat it promptly, and thus to reduce its duration. If the same effect could be achieved for exacerbations of a concomitant disease, the effect of self-management could be amplified and thus become statistically significant.

The reduction in hospitalisations is an important result that should be explored further. Rehospitalisation due to an exacerbation of COPD is common within 30-60 days, particularly in patients with multimorbidity (J. Alqahtani, M.C. Njoku, B. Wimmer B, et al.; unpublished data). Patients recruited to the study after hospitalisation due to a COPD exacerbation have a reduced frequency of rehospitalisation when following an action plan, such as one with video-assisted telehealth rehabilitation [15]. Thus, LENFERINK et al. [10] might consider conducting an even shorter study with patients discharged after hospitalisation due to an exacerbation of COPD or one of the concomitant chronic diseases, and powering the study on hospital readmission. Furthermore, the self-management plan could be revised to include a case manager for each patient.

We congratulate the authors for conducting a rigorous and elegant study, and we agree that their approach opens new avenues for investigating the role of self-management interventions, including specific tools for addressing exacerbations and concomitant chronic diseases in patients with COPD and multimorbidities. However, while we share the authors' optimism, it is important to remember that positive results for secondary outcomes obtained in studies negative for the primary outcome cannot be used to draw conclusions or, more importantly, to make recommendations. The authors' results can be used to generate hypotheses and to plan further studies [16].

Conflict of interest: L.E.G.W. Vanfleteren reports personal fees from AstraZeneca, Chiesi, Pulmonx, GlaxoSmithKline and Novartis, outside the submitted work. L.M. Fabbri reports personal fees and non-financial support from AstraZeneca, GSK, Novartis, Menarini, Boehringer Ingelheim, Zambon and Pearl Therapeutics, grants, personal fees and non-financial support from Chiesi, and non-financial support from Dompe, outside the submitted work. 


\section{References}

1 Zwerink M, Brusse-Keizer M, van der Valk PD, et al. Self management for patients with chronic obstructive pulmonary disease. Cochrane Database Syst Rev 2014; 3: CD002990.

2 Lenferink A, Brusse-Keizer M, van der Valk PD, et al. Self-management interventions including action plans for exacerbations versus usual care in patients with chronic obstructive pulmonary disease. Cochrane Database Syst Rev 2017; 8: CD011682.

3 The Global Initiative for Chronic Obstructive Lung Disease (GOLD). 2019 Global Strategy for Prevention, Diagnosis and Management of COPD. www.goldcopd.org Date last accessed: 16 September 2019.

4 Ferrone M, Masciantonio MG, Malus N, et al. The impact of integrated disease management in high-risk COPD patients in primary care. NPJ Prim Care Respir Med 2019; 29: 8.

5 Fan VS, Gaziano JM, Lew R, et al. A comprehensive care management program to prevent chronic obstructive pulmonary disease hospitalizations: a randomized, controlled trial. Ann Intern Med 2012; 156: 673-683.

6 National Institute for Health and Care Excellence: Clinical Guidelines. Multimorbidity: Assessment, Prioritisation and Management of Care for People with Commonly Occurring Multimorbidity. London, National Institute for Health and Care Excellence, 2016.

7 Franssen FME, Vanfleteren LEGW. Differential diagnosis and impact of cardiovascular comorbidities and pulmonary embolism during COPD exacerbations. In: Burgel PR, Contoli M, López-Campos JL, eds. ERS Monograph: Acute Exacerbations of Pulmonary Diseases. Sheffield, European Respiratory Society, 2017; pp. $114-128$

8 Vanfleteren LE, Spruit MA, Franssen FM. Tailoring the approach to multimorbidity in adults with respiratory disease: the NICE guideline. Eur Respir J 2017; 49: 1601696.

9 Salisbury C, Man MS, Bower P, et al. Management of multimorbidity using a patient-centred care model: a pragmatic cluster-randomised trial of the 3D approach. Lancet 2018; 392: 41-50.

10 Lenferink A, van der Palen J, van der Valk PDLPM, et al. Exacerbation action plans for patients with COPD and comorbidities: a randomised controlled trial. Eur Respir J 2019; 54: 1802134

11 Kessler R, Casan-Clara P, Koehler D, et al. COMET: a multicomponent home-based disease-management programme versus routine care in severe COPD. Eur Respir J 2018; 51: 1701612.

12 Rose L, Istanboulian L, Carriere L, et al. Program of Integrated Care for Patients with Chronic Obstructive Pulmonary Disease and Multiple Comorbidities (PIC COPD ${ }^{+}$): a randomised controlled trial. Eur Respir J 2018; 51: 1701567.

13 Lenferink A, Frith P, van der Valk P, et al. A self-management approach using self-initiated action plans for symptoms with ongoing nurse support in patients with chronic obstructive pulmonary disease (COPD) and comorbidities: the COPE-III study protocol. Contemp Clin Trials 2013; 36: 81-89.

14 Rea F, Calusi G, Franchi M, et al. Adherence of elderly patients with cardiovascular disease to statins and the risk of exacerbation of chronic obstructive pulmonary disease: evidence from an Italian real-world investigation. Drugs Aging 2018; 35: 1099-1108.

15 Bhatt SP, Patel SB, Anderson EM, et al. Video telehealth pulmonary rehabilitation intervention in chronic obstructive pulmonary disease reduces 30-day readmissions. Am J Respir Crit Care Med 2019; 200: 511-513.

16 Vanfleteren L, Ullman A, Fabbri LM. Time for a longer and better life for patients with COPD. Eur Respir J 2018; 51: 1702569. 